\title{
Induced anisotropies in NiCo obliquely deposited films and their effect on magnetic domains
}

\author{
H. Aitlamine, L. Abelmann, and I. B. Puchalska \\ C.N.R.S., Laboratoire de Magnétisme et Matériaux Magnétiques, 92195 Meudon Cedex, France
}

(Received 3 January 1991; accepted for publication 24 September 1991)

Oblique and in-plane anisotropies in obliquely evaporated NiCo thin films were investigated in order to understand their origin. All the compositions studied clearly show the effect of columnar grain morphology coupled with some intrinsic factors such as magnetostriction and crystallinity. Energy calculations are undertaken to explain the effect of these anisotropies on domain structures and the existence of strong and weak stripe domains depending on the composition and incidence angle.

\section{INTRODUCTION}

Thin films are the most promising media for high-density information storage. This is due to their continuous structure and the fact that they exhihit a high remanent magnetic induction with high coercitivity.

In longitudinal recording the NiCo alloy is one of the primary materials used. The films are obliquely evaporated in order to induce an oblique anisotropy. ${ }^{1,2}$ The magnetic properties of the media can be controlled by the NiCo composition, the angle of incidence (with respect to the film normal), the degree of oxidation, and the thickness of the magnetic layer. Among the most studied properties are the induced anisotropies which are related to the particular grain texture. These anisotropies are (Fig. 1) (i) the oblique anisotropy $K_{o}$, which is related to the crystallites which grow in the form of columns (these columns are parallel to the plane of deposition and are inclined at an angle to the film normal), and (ii) the planar anisotropy $K_{p}$, which is related to the ordering of these columns in chains perpendicular to the deposition plane.

These anisotropies have been studied for various materials, ${ }^{1-4}$ and their physical origin has mainly been attributed to magnetostatic effects (shape anisotropy) related to the microtexture. Nevertheless, several authors ${ }^{4-6}$ also underline the importance of the crystalline anisotropy contribution and also the anisotropy due to the internal stress in this type of film. The first purpose of this paper is to look further into the origin of the anisotropy, analyzing the experimental results obtained for NiCo films using a torque balance.

The purpuse of the second part of this work is a theoretical study of stripe-domain structures related to the existence of oblique anisotropy. These structures consist of regular, periodic domains where the magnetization is out of the film plane with flux closure domains at the film surface. Depending on the magnetization direction of the adjacent domains, there are two kinds of stripe domains which are either strong stripe domains or weak stripe domains $^{7}$ (their specific domain structures will be discussed later on). Strong stripe domains were first observed in Permalloy ${ }^{7,8}$ and have also been observed in $\mathrm{Ni}_{0.2} \mathrm{Co}_{0.8}$ films by Puchalska et al. ${ }^{9}$ who suggested that this structure could be used in magnetic recording.
A detailed study of stripe domains in NiCo films was given in our previous paper. ${ }^{10}$ We showed that the existence of these structures is subject to the conditions of the film thickness $D$ and the angle of deposition $\alpha$, which must exceed critical values $D_{c}$ and $\alpha_{c}$. We also found that the nature of the observable stripe domains (i.e., whether weak and/or strong stripes are observed) and the values of $D_{c}$ and $\alpha_{c}$ depend on the film composition. In order to explain these results, we study the effect of the induced anisotropies on the existence of stripe domains.

\section{EXPERIMENT}

Thin films of $\mathrm{Ni}_{x} \mathrm{Co}_{1-x}$ with $x=0.2,0.5$, and 0.8 were obliquely evaporated onto Corning 7059 glass substrates from bulk alloy material. Since the vapor pressures of Co and $\mathrm{Ni}$ are almost identical, the final composition of the film is close to the source material composition, which is determined by weighing each component. Occasional microprobe analysis showed that the film composition differs from the alloy composition by a maximum of $2 \%$.

The deposition geometry is shown in Fig. 1(a). The angle of deposition $\alpha$ was varied from $0^{\circ}$ to $60^{\circ}$ with the source-substrate distance fixed at $13 \mathrm{~cm}$. The thickness of the studied films is ranged from 0.1 to $1.3 \mu \mathrm{m}$ with a deposition rate of $10-100 \AA / \mathrm{min}$. Depending on the film composition and thickness, the residual pressure varies from $1 \times 10^{-6}$ to $5 \times 10^{-5}$ Torr, and the temperature of

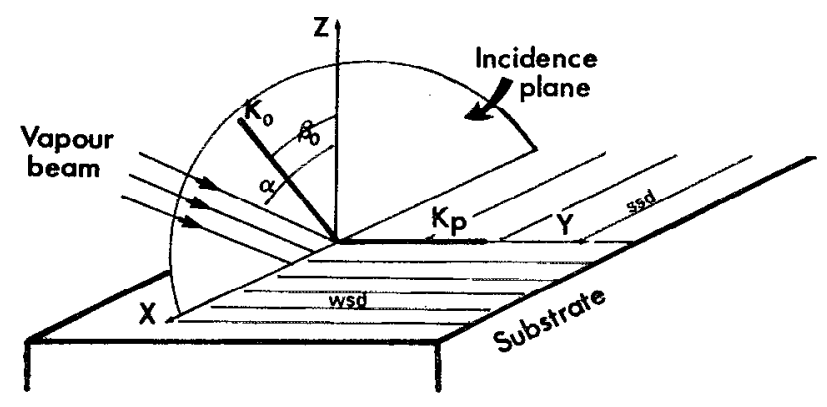

FIG. 1. Schematic representation of oblique deposition and directions of anisotropy axes, weak (WSD) and strong (SSD) stripe domains. 
the holder on which the substrates are mounted varies from room temperature up to $100{ }^{\circ} \mathrm{C}$. (The relation between substrate and holder temperatures is unknown, but the substrate temperature can be expected to be higher.) However, we ensure that the elaboration parameters are within the range that gives crystallite growth in forms of columns, inclined at an angle $\beta_{\mathrm{col}}$, which follows the tangent rule $\left[2 \tan \left(\beta_{\mathrm{col}}\right)=\tan (\alpha)\right]$, as reported earlier. ${ }^{11-13}$

The magnetic properties of the samples were measured using a vibrating-sample magnetometer and a torque balance. The magnetic domains were observed using either a digitally enhanced Kerr-effect microscope ${ }^{14}$ or a high-resolution Bitter technique.

\section{THE ORIGIN OF THE INDUCED ANISOTROPIES}

\section{A. Principle of anisotropies measurements}

The oblique anisotropy $K_{o}$ and the position of the anisotropy axis $\beta_{o}$ are obtained by torque measurements. The torque is measured under the application of a rotating field in the incidence plane. The magnitude of the maximum field is $11 \mathrm{kOe}$. The values of $K_{o}$ and $\beta_{o}$ are deduced from the following relations: ${ }^{3}$

$$
\tan \left(2 \beta_{o}\right)=\frac{L_{1} \sin 2 \Phi}{2 \pi M_{s}^{2}-L_{1} \cos 2 \Phi}
$$

and

$$
K_{o}=L_{1} \frac{\sin 2 \Phi}{\sin 2 \beta_{o}},
$$

where $\Phi$ is the angle of zero torque and $L_{1}$ is the maximum value of the measured torque which is reached at $\Phi+\pi / 2$. The angle $\Phi$ indicales the position of an effective out-ofplane easy axis resulting from the competition between the oblique anisotropy $K_{o}$ and the demagnetizing factor $2 \pi M_{s}^{2}$

To determine the in-plane anisotropy $K_{p}$, the field is applied in the film plane and rotates around the normal to the film. The equilibrium condition leads to the relation

$$
K_{p}=L_{2}+K_{o} \sin \beta_{o}
$$

where $L_{2}$ is the maximum torque measured.

\section{B. Results and discussions}

\section{1. $\mathrm{Ni}_{0.8} \mathrm{CO}_{0.2}$ films (magnetoelastic contribution)}

We measured the value and the direction of the oblique anisotropy $K_{o}$ as a function of the incidence angle and for different film thicknesses [Figs. 2(a) and 2(b)]. The magnitude of $K_{o}$ appears to be independent of the film thickness, whereas its variation with the incidence angle is more significant, giving an understanding of the origin of anisotropy.

Although the position of the oblique anisotropy axis corresponds to the main axis of the column' [Fig. 2(b), the measured anisotropy does not vary as a typical shape anisotropy connected with columnar grain morphology. A
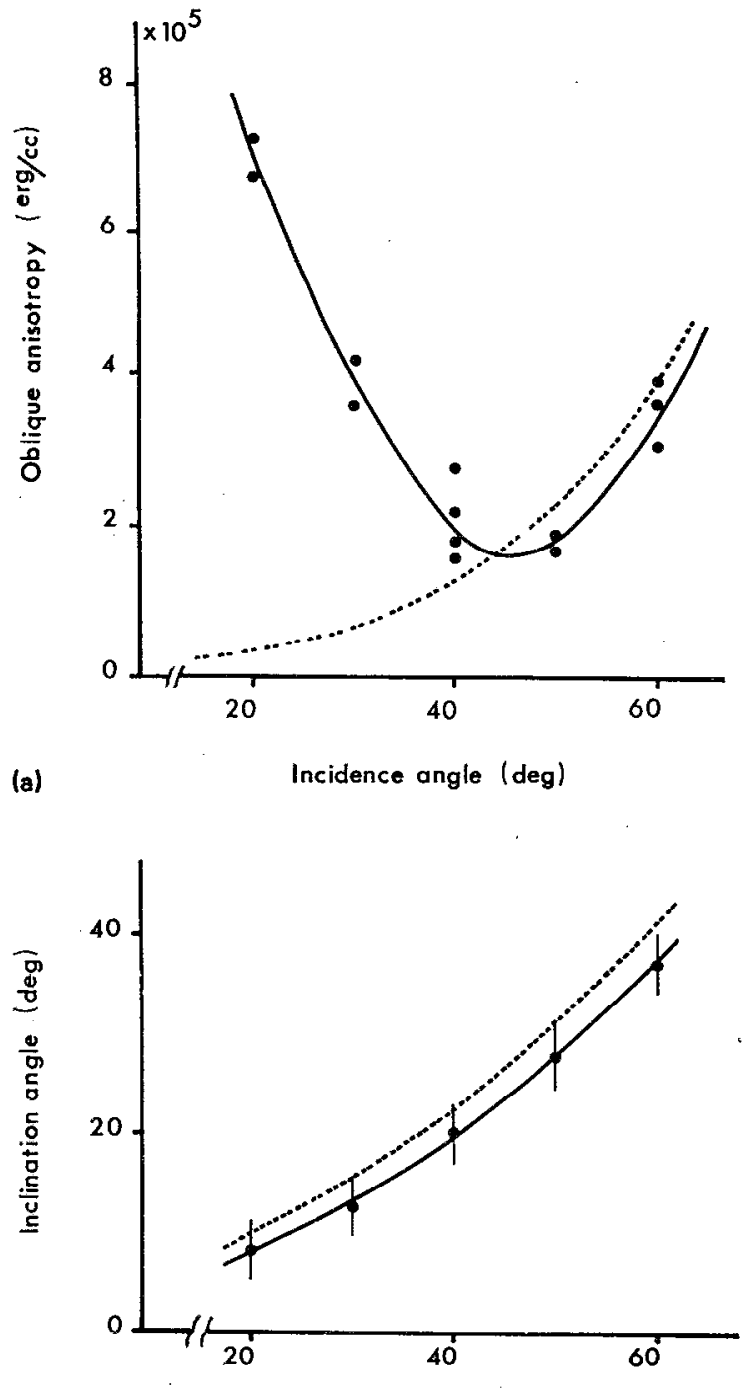

(b)

$$
\text { Incidence angle (deg) }
$$

FIG. 2. Oblique anisotropy vs incidence angle for $\mathrm{Ni}_{0.8} \mathrm{Co}_{0.2}$ films. Several film thicknesses per incidence angle were measured. The dotted line represents the variation of oblique anisotropy expected from columnar shape. (b) Inclination of the oblique anisotropy axis $\left(\beta_{o}\right)$ measured as a function of incidence angle for $\mathrm{Ni}_{0.8} \mathrm{Co}_{0.2}$ films. The dotted line indicates the inclination of the columnar texture calculated by the tangent rule. ${ }^{16}$

simple, but for our purposes effective, description of the shape anisotropy caused by a columnar structure has been given by Hara. ${ }^{3}$ (For a more detailed description, see Münster ${ }^{15}$.) Hara assumes the material to be composed of a matrix of ferromagnetic parallelepipeds separated by nonmagnetic space. The anisotropy constants are determined by calculating the demagnetizing fields. The value of the shape anisotropy was found to depend on $M_{s}$ and the ratios (measured in three directions) between the width of the parallelepipeds and distance in between them. Hara verified his calculations for obliquely evaporated $\mathrm{Fe}$ films. We assume the ratios mentioned above to be equal for $\mathrm{Fe}$ and $\mathrm{Ni}_{0.8} \mathrm{Co}_{0.2}$ and therefore expect the value of the shape anisotropy to vary with incidence angle as represented by the dotted line in Fig. 2(a). The discrepancy between the measured and calculated values clearly indicates that an- 


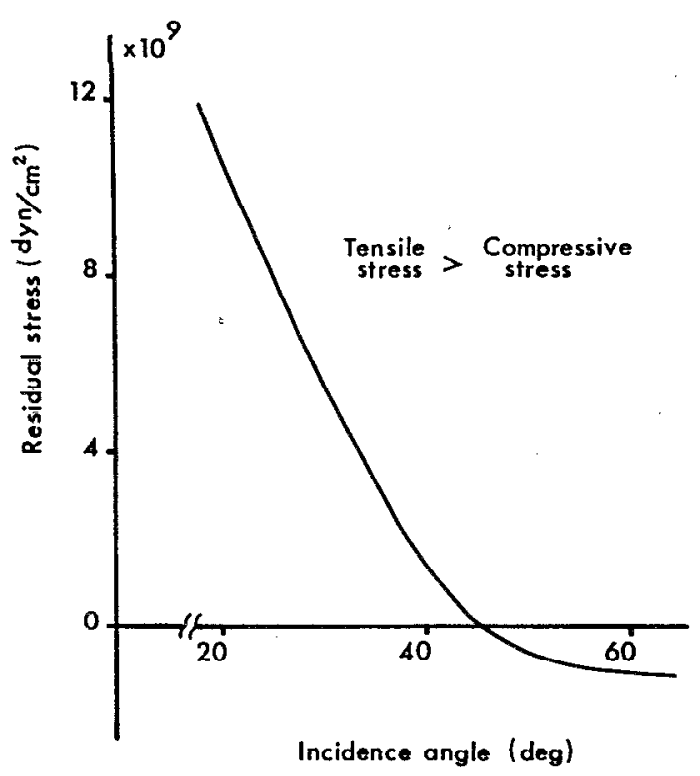

FIG. 3. Stress in $\mathrm{Ni}_{0.8} \mathrm{Co}_{0.2}$ films vs incidence angle, deduced from anisotropy measurements.

other mechanism contributes to the total anisotropy besides the shape of columns. As the magnetocrystalline anisotropy of this alloy is very small, ${ }^{17,18}$ we believe that residual stresses are mainly responsible for increasing the total anisotropy at small angles of deposition. For highincidence angles, the stress must decrease and the shape anisotropy predominates. The measured oblique anisotropy can be expressed as a sum of shape and stress anisotropies:

$$
K_{o}=K_{\text {shape }}+K_{\text {stress }}
$$

From this formula and using the average magnetostriction of the bulk alloy, ${ }^{19}$ we deduce the variation of the residual stress versus the incidence angle (Fig. 3 ). Here we assume an anisotropic stress acting on the columns perpendicularly to their axis. We find that there should be a tensile stress for low-incidence angles. This stress should decrease with increasing incidence angle. (The stress in the films investigated has, however, not been measured, but the values estimated are in the range of stresses usually measured in metallic films.)

This interpretation of the origin of oblique anisotropy in $\mathrm{Ni}_{0.8} \mathrm{Co}_{0.2}$ leads us to discuss the origin of residual stress in obliquely evaporated films. Stress in thin metallic films has been observed and measured by many investigators, ${ }^{20,22}$ who reported either tensile or compressive stress. It has been also shown that stress in obliquely deposited films is anisotropic. ${ }^{23,24}$ Although the mechanisms generating stress in thin films are not completely understood, we suggest, however, that the stress in our films is caused by two competing mechanisms. On the one hand, a tensile stress is produced by the tendency of crystallites to coalescence by diffusion while the film is growing. On the other hand, as the temperature decreases below the crystallization temperature, the diffusion is no longer

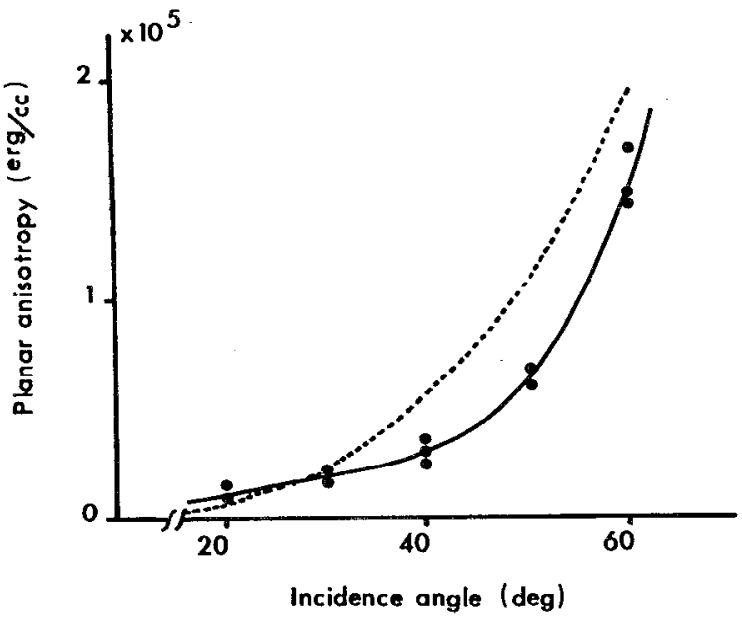

FIG. 4. In-plane anisotropy $K_{p}$ in $\mathrm{Ni}_{0.8} \mathrm{Co}_{0.2}$ films vs incidence angle. The dotted line represents the variation of $K_{p}$ estimated from the shape of columns elongated in chains perpendicular to the incidence plane.

favored and the intercolumnar environment will cause a compressive stress. ${ }^{20}$ The balance between the two mechanisms, which act simultaneously, will determine whether the resulting stress in the film is compressive or tensile. For low-incidence angles, the tensile stress predominates because the columns are very close together. For high-incidence angles, as the intercolumnar space increases, the compressive stress increases and the tensile stress decreases so giving a decrease in the total stress.

For the in-plane anisotropy $K_{p}$, in-plane torque measurements show that the film is almost isotropic. This means that the uniaxial in-plane anisotropy is balanced by the in-plane contribution of $K_{o}$ so that

$$
K_{p} \approx K_{o} \sin ^{2} \beta_{o} \text {. }
$$

The variation of $K_{p}$ versus the incidence angle (Fig. 4) correlates with the calculated shape anisotropy resulting from the chains of columns perpendicular to the incidence plane (dotted line). The calculation is an analog to that of $K_{o}$ [Fig. 2(a)].

\section{2. $\mathrm{Ni}_{0.2} \mathrm{Co}_{0.8}$ films (magnetocrystalline contribution)}

This alloy gives evidence of another contribution to the oblique anisotropy. This is the magnetocrystalline anisotropy connected with the hexagonal structure of $\mathrm{Co}$ and the presence of a $c$-axis texture at high angles of deposition. ${ }^{4}$ Table I shows the measured values of $K_{o}, K_{p}$, and $\beta_{o}$ for different samples elaborated at $60^{\circ}$.

As for $\mathrm{Ni}_{0.8} \mathrm{Co}_{0.2}$, there is no obvious behavior of the anisotropies with the thickness of the film. We note, however, that the oblique easy axis is inclined at angle of $20^{\circ}$ to the normal, whereas we would expect, by the tangent rule, an inclination of the columns of $41^{\circ}$. Also, the measured anisotropy is of the order of $2 \times 10^{6} \mathrm{erg} / \mathrm{cc}$, and from shape anisotropy alone, we would expect an oblique anisotropy of $1.3 \times 10^{6} \mathrm{erg} / \mathrm{cc}$. This clearly shows the influence of hcp 
TABLE I. Anisotropy measurements of $\mathrm{Ni}_{0.2} \mathrm{Co}_{0.8}$ films for various thicknesses and an incidence angle $\alpha=60^{\circ}$.

\begin{tabular}{rccc}
\hline \hline $\begin{array}{c}\text { Thickness } \\
\mathrm{D}(\AA)\end{array}$ & $\begin{array}{c}K_{o} \\
\left(\times 10^{5} \mathrm{erg} / \mathrm{cc}\right)\end{array}$ & $\begin{array}{c}\beta_{o} \\
(\mathrm{deg})\end{array}$ & $\begin{array}{c}K_{p} \\
\left(\times 10^{5} \mathrm{erg} / \mathrm{cc}\right)\end{array}$ \\
\hline 1000 & 18 & 22 & 5.68 \\
1300 & 22.2 & 19 & 3.25 \\
5000 & 13.4 & 19 & 3.27 \\
11000 & 28.2 & 22 & 13.80 \\
\hline
\end{tabular}

texture which affects the orientation and value of the shape anisotropy, giving the observed oblique anisotropy. The presence of this texture, the $c$ axis of which does not coincide with the main axis of the columns, was shown by Ho, Gau, and Thomas, ${ }^{4}$ who suggested that the magnetocrystalline anisotropy of $\mathrm{Ni}_{0.2} \mathrm{Co}_{0.8}$ would contribute to the total anisotropy. We can calculate this contribution and the main orientation of the $c$ axis using our experimental measurement of anisotropy and the calculated value of shape anisotropy. To do this, we consider a single domain model where the sample is saturated in the incidence plane.

The torque which acts on the magnetization vector and results from the competition between shape anisotropy $\left(K_{\mathrm{col}}, \beta_{\mathrm{col}}\right)$ and magnetocrystalline anisotropy $\left(K_{\mathrm{cr}}, \beta_{\mathrm{cr}}\right)$, aligned parallel to the $c$ axis, is equivalent to the torque resulting from the effective anisotropy $\left(K_{o}, \beta_{o}\right)$, which we measure (Fig. 5). The relation which describes this equivalence is

$$
\begin{gathered}
K_{\mathrm{col}} \sin 2\left(\theta-\beta_{\mathrm{col}}\right)+K_{\mathrm{cr}} \sin 2\left(\theta-\beta_{\mathrm{cr}}\right) \\
=K_{o} \sin 2\left(\theta-\beta_{o}\right),
\end{gathered}
$$

where $\theta$ is the angle between the magnetization and normal to the film. Since this relation is valid for every $\theta$, we can deduce the following expressions for $\beta_{\mathrm{cr}}$ and $K_{\mathrm{cr}}$ :

$$
\begin{aligned}
& \tan \left(2 \beta_{\mathrm{cr}}\right)=\frac{K_{o} \sin 2 \beta_{o}-K_{\mathrm{col}} \sin 2 \beta_{\mathrm{col}}}{K_{o} \cos 2 \beta_{o}-K_{\mathrm{col}} \cos 2 \beta_{\mathrm{col}}}, \\
& K_{\mathrm{cr}}=\frac{K_{o} \sin 2 \beta_{o}-K_{\mathrm{col}} \sin 2 \beta_{\mathrm{col}}}{\sin 2 \beta_{\mathrm{cr}}} .
\end{aligned}
$$

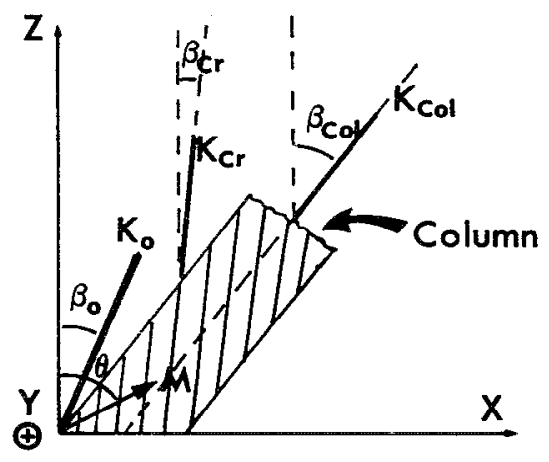

FIG. 5. Schematic representation of measured oblique anisotropy $\left(K_{o}, \beta_{o}\right)$ resulting from the competition between magnetocrystalline anisotropy $\left(K_{\mathrm{cr}}, \beta_{\mathrm{cr}}\right)$ and shape anisotropy $\left(K_{\mathrm{col}}, \beta_{\mathrm{col}}\right)$ in $\mathrm{Ni}_{0.2} \mathrm{Co}_{0.8}$ films.

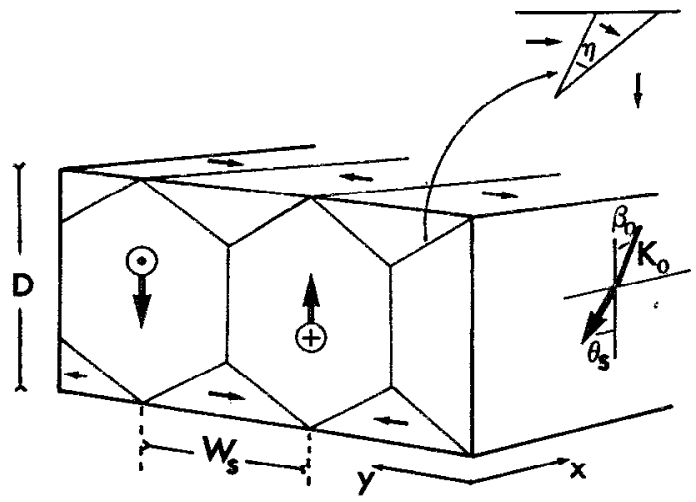

FIG. 6. Magnetization distribution in strong stripe domains proposed by Hara (Ref. 3). The inset shows the transition region between a basic domain and a closure domain.

Using the average of the measured values of $\left(K_{o}, \beta_{o}\right)$ and the calculated values of $\left(K_{\mathrm{col}}, \beta_{\mathrm{col}}\right)$, we obtain $\beta_{\mathrm{cr}}$ in the range of $6^{\circ}-10^{\circ}$ and $K_{\mathrm{cr}} \approx 1.5 \times 10^{6} \mathrm{erg} / \mathrm{cc}$. The $c$ axis tends to be perpendicular to the film plane.

\section{INFLUENCE OF THE INDUCED ANISOTROPIES ON MAGNETIZATION DISTRIBUTION AND DOMAIN STRUCTURES}

The presence of oblique and in-plane anisotropies causes an unusual magnetic process (hysteresis) due to a particular distribution of magnetization in the film. This distribution is characterized by the presence of weak and strong stripe-domain structures. The magnetization in these domains is out of the film plane, along the easy axis, with flux closure domains at the surface. The weak stripe domains are elongated perpendicular to the vapor incidence direction, whereas the strong stripe domains are elongated parallel to the vapor incidence plane (Fig. 1). The existence of these structures in NiCo films was reported previously. ${ }^{9,10}$ However the existence of stripe domains depends on the incidence angle, which must exceed a critical value, and their nature (weak or strong stripes) depends on the film composition: In the $\mathrm{Ni}_{0.8} \mathrm{Co}_{0.2}$ films, both structures can coexist, whereas in $\mathrm{Ni}_{0.5} \mathrm{Co}_{0.5}$ and $\mathrm{Ni}_{0.2} \mathrm{Co}_{0.8}$ films, only the strong stripes are observed.

In this section, we attempt to clarify the effects of the composition and angle of deposition on the existence of the stripe domains. We will show that these effects are, in fact, due to the anisotropies induced. The interpretation is based on elementary calculations of the stripe-domain energy compared with the in-plane single-domain energy. We will use "SSD" to indicate strong stripe domains, and "WSD" to indicate weak stripe domains.

\section{A. Theory of stripe-domain energy}

\section{Energy of SSD structure}

For these calculations we used the basic model of the SSD proposed by Hara. ${ }^{3}$ This stray-field-free model (Fig. 6) does not completely contradict the micromagnetic cal- 
culation presented recently by Labrune and $\mathrm{Miltat}^{25}$ and can be considered as a first-order approximation of the real distribution of the magnetization in thick films $\left(D>D_{c}\right)$.

The equilibrium parameters are the angle $\theta_{s}$, which represents the inclination of the magnetization in the basic domain with respect to the normal of the film, and the half-period $W_{s}$. The central wall between two adjacent basic domains is a $180^{\circ}$ Bloch wall. The energy of the zone of transition between basic and closure domains is calculated as a wall energy using the method described by Hubert. ${ }^{26}$ For simplicity of calculation, it was assumed that in the center of the transition zone the magnetization is perpendicular to the wall [and so has no $x$ component (Fig. 1)]. Micromagnetic calculations, however, show that the magnetization follows a more stray-field-free path. ${ }^{25}$ Our simplification gives cause to a transition-zone energy which is only $10 \%-22 \%$ higher than stray-field-free model, and so the influence on the total domain energy will be small. The optimization of the transition zone with respect to the aperture angle $\eta$, which defines the zone of transition, gives the following expression:

$$
\sigma_{\mathrm{TS}}=\Omega L(A \Delta K / 2)^{1 / 2},
$$

where $A$ is the exchange constant, $L$ is the transition wall length, and $\Delta K$ is the anisotropy energy difference between a point in the wall center and a point in the basic or closure domain. $\Omega$ is the total rotation angle of the magnetization when moving from the oblique position in the basic domain to the in-plane position in the closure domain, following a stray-field-free path.

The total energy of the structure pcr unit area of the film is then given by

$$
\begin{aligned}
E\left(\theta_{s} W_{s}\right)= & {\left[K_{p}-K_{o} \cos ^{2}\left(\theta_{s}-\beta_{o}\right)\right]\left(D-W_{s} \cos \theta_{s} / 2\right) } \\
& +\sigma_{B}\left(D / W_{s}-\cos \theta_{s}\right)+4 \sigma_{\mathrm{TS}} / W_{s} .
\end{aligned}
$$

The first term is the anisotropy energy, and the second and third terms are related to the energies of the central and transition walls respectively. $\sigma_{B}$ is the energy density of $180^{\circ}$ Bloch wall.

The minimum thickness of the film required here corresponds to the thickness for which the central wall length vanishes:

$$
D_{c}=\frac{2 \sigma_{B} \cos \theta_{s}}{K_{o} \cos ^{2}\left(\theta_{s}-\beta_{o}\right)-K_{p}} .
$$

\section{SSD under the application of a transverse field}

Now we consider the energy of the SSD with an applied field in the film plane and perpendicular to the direction of their elongation. From experimental observations ${ }^{10}$ [Fig. 7(a)], we can deduce the effect of this field on the domain structure [Fig. 7(b)].

At the surface, the closure domain favorable to the field expands while the other one shrinks. The closure domains are characterized by (i) their widths $W_{1}$ and $W_{2}$, the total periodicity $2 W_{s}$ remaining constant
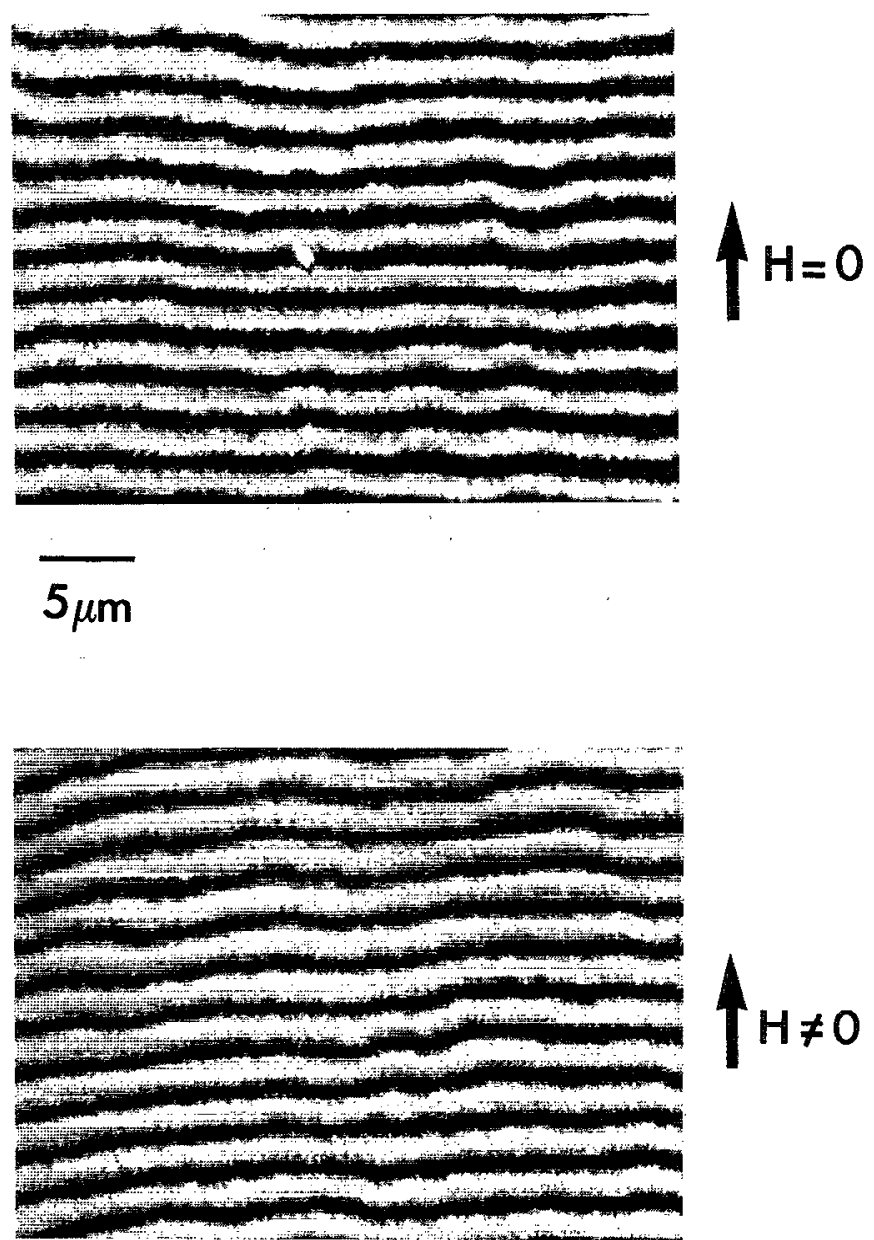

(a)

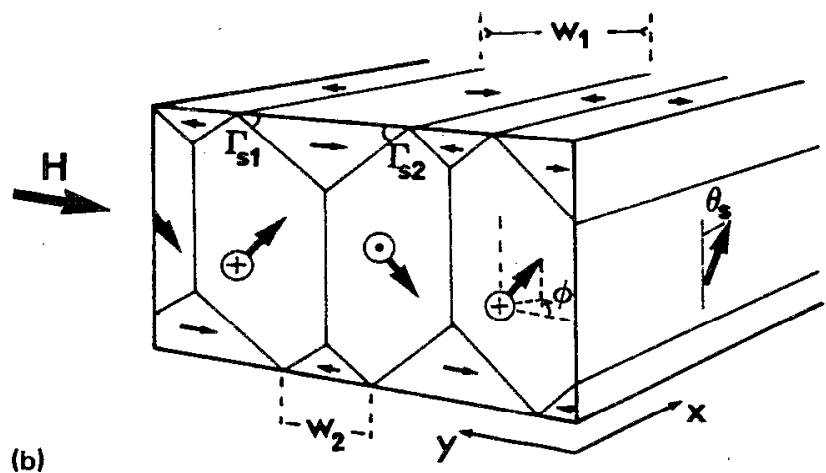

FIG. 7. (a) Strong stripe domains in a $\mathrm{Ni}_{0.5} \mathrm{Co}_{0.5}$ film $(\alpha=50, D=1.3$ $\mu \mathrm{m}$ ) observed by longitudinal Kerr-effect microscopy. (b) Proposed magnetization distribution of strong stripe domains under application of an external field.

$2 W_{s}=W_{1}+W_{2}$; (ii) $\Gamma_{s 1}$ and $\Gamma_{s 2}$, the inclination angles of transition walls; and (iii) the energies $\sigma_{\mathrm{TS} 1}$ and $\sigma_{\mathrm{TS} 2}$ of these walls.

Since the field is applied perpendicular to the central wall, the magnetization in the basic domains turns within the plane formed by its initial inclination and the direction of the field. The total energy per unit area of the film is then 


$$
\begin{aligned}
E\left(\theta_{s}, W_{1}, H, \phi\right)= & \left(D-\frac{W_{1}^{2} \tan \Gamma_{s 1}+W_{2}^{2} \tan \Gamma_{s 2}}{4 W_{s}}\right)\left[K_{p}-K_{o} \cos ^{2}\left(\theta_{s}-\beta_{o}\right)\right] \sin ^{2} \phi \\
& +\left(D-\frac{W_{1} \tan \Gamma_{s 1}+W_{2} \tan \Gamma_{s 2}}{2}\right) \frac{\sigma_{B} \sin ^{2} \phi}{W_{s}}+\frac{2\left(\sigma_{\mathrm{TS} 1}+\sigma_{\mathrm{TS} 2}\right)}{W_{s}} \\
& +\frac{H M_{s}}{W_{s}}\left[\left(D-W_{1}^{2} / 4 W_{s}\right)(1-\cos \phi)+(1+\cos \phi) W_{2}^{2} / 4 W_{s}\right]
\end{aligned}
$$

where $\phi$ is the angle between the in-plane component of magnetization vector and the $y$ axis. At $\phi=\pi / 2$ the undisturbed ( $H=0)$ SSD structure has returned [Fig. 7(b)].

\section{Energy of WSD structure}

We consider here the WSD structure which may result from the application of an external field perpendicular to the SSD structure as we will show later. The model of WSD is represented in Fig. 8.

The structure is perpendicular to the incidence plane which, after Hubert, ${ }^{27}$ gives inclined central walls because of the inclination of the anisotropy. The starting point of the treatment is to assume a slight oscillation of the magnetization in the surface plane for closure domains and in the plane formed by the oblique anisotropy axis and direction of stripes for basic domains. To simplify the problem, we represent this oscillation by the same angle $\theta_{w}$ for both domains. $\theta_{w}$ increases with increasing field applied along the stripes. The transition walls (TWs) are characterized by their inclination angles $\Gamma_{w 1}$ and $\Gamma_{w 2}$ $\left(\Gamma_{w 1}+\Gamma_{w 2}=\pi / 2\right)$ and by their energies $\sigma_{\mathrm{TW} 1}$ and $\sigma_{\mathrm{TW} 2}$. The total energy per unit area of the film is

$$
\begin{aligned}
E\left(\theta_{w}, W_{w}, H\right)= & \cos 2 \theta_{w}\left(D\left(K_{p}-K_{o}\right)+K_{o} W_{w} \tau \cos ^{2} \beta_{o}\right) \\
& +\sigma_{B} \frac{\cos ^{2} \theta_{s}}{\cos \beta_{o}}\left(D / W_{w}-2 \tau\right) \\
& +2\left(\sigma_{\mathrm{TW} 1}+\sigma_{\mathrm{TW} 2}\right)+H M_{s}(1-\sin \phi),
\end{aligned}
$$

with

$$
\tau=\frac{\tan \Gamma_{w 1} \tan \Gamma_{w 2}}{\tan \Gamma_{w 1}+\tan \Gamma_{w 2}}
$$

$W_{w}$ is the half-period of the stripes and is independent of the applied field.

\section{B. Effect of the composition on the nature of stripes}

We discuss here the transition from SSD to WSD structure. This transition depends on the film composition and may occur under the application of a given field between zero and the saturation field. Figure 9(a) shows an experimental observation of this transition, which we schematize in Fig. $9(\mathrm{~b})$.

Figures 10(a) and 10(b) show the numerical computations of the SSD and WSD energies at zero and applied fields for which the transition should occur. In these figures we vary the ratio $K_{p} / K_{o}$, where $K_{o}$ is kept constant and equal to the experimental value measured for each composition at a given angle of deposition, which is $40^{\circ}$ for $\mathrm{Ni}_{0.8} \mathrm{Co}_{0.2}$ and $\mathrm{Ni}_{0.5} \mathrm{Co}_{0.5}$ films and $60^{\circ}$ for the $\mathrm{Ni}_{0.2} \mathrm{Co}_{0.8}$ films. These angles are chosen to give the same inclination of the oblique anisotropy axis, which is $\beta_{0}=20^{\circ}$. The thickness is fixed at $0.5 \mu \mathrm{m}$ for all compositions.

At zero field we can see that the SSD structure has a lower energy than the WSD structure. This is in agreement with experimental observations since we observe only regular strong stripe domains when the sample is carefully demagnetized along the incidence plane. An increase of $K_{p}$ does not favor either the WSD or SSD structure: For the WSD structure, the energy approaches the planar domain energy as $K_{p}$ increases. For the SSD structure, even if the energy remains favorable with increasing $K_{p}$, the structure no longer exists, however, because of the critical thickness defined in (11). Thus we can define a critical value of the ratio $K_{p} / K_{o}$ above in which the strong stripe does not exist. This critical ratio depends on the thickness of the film and the angle of deposition:

$$
\left(K_{p} / K_{o}\right)_{c}=1-\frac{8 \cos \beta_{o}\left(A / K_{o}\right)^{1 / 2}}{D} .
$$

We will discuss the relation between this critical ratio and the angle of deposition in the next section.

In the presence of an applied field and up to a certain value of $K_{p} / K_{o}$, the WSD structure is favored compared to the SSD structure because its energy is lower. A transition between the two structures is then possible. Above this value of $K_{p} / K_{o}$, the SSD remains stable even if the field is increased, and no transition will occur. Table II summarizes for the three compositions the ranges of the ratio

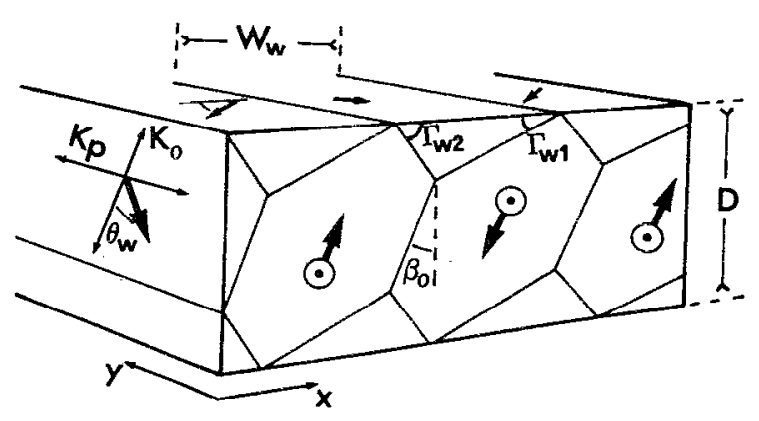

FIG. 8. Model of weak stripe domains perpendicular to the incidence plane [after Hubert (Ref. 27)]. 


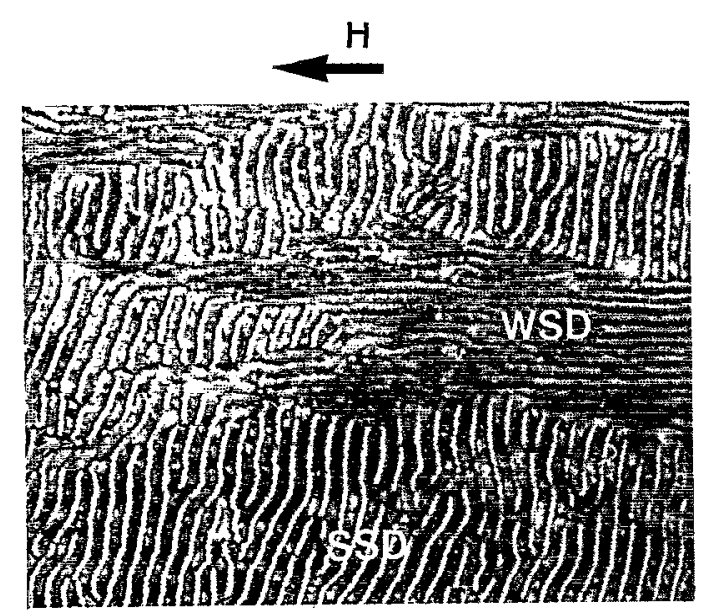

$\overline{5 \mu \mathrm{m}}$

(a)

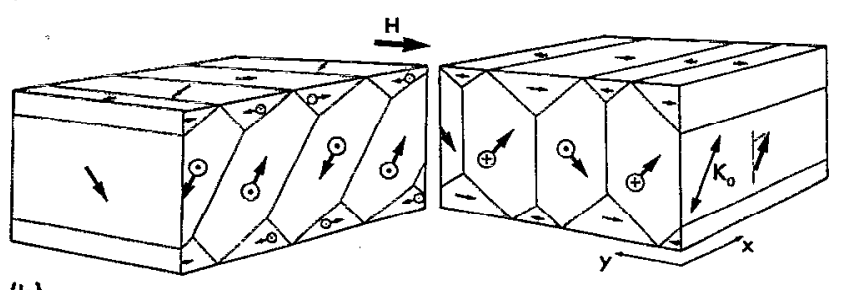

(b)

FIG. 9. (a) Dry Bitter pattern of the transition from strong to weak stripe structure in a $\mathrm{Ni}_{0.8} \mathrm{CO}_{0.2}$ film $\left(\alpha=50^{\circ}, D=0.8 \mu \mathrm{m}\right)$, after the application of an external field perpendicular to the strong stripe structure. (b) Schematization of the transition represented in (a).

$K_{p} / K_{o}$, where the transition is possible, and also the measured values of this ratio. We deduce from this table that the weak stripe structure cannot exist in cobalt-rich films evaporated at oblique incidence, since they display high values of $K_{p} / K_{o}$. This interpretation is in good agreement with our experimental observations of stripe domains. ${ }^{10}$

\section{Effect of the incidence angle (the critical angle)}

The existence of a critical angle of deposition below which the SSD structure does not exist was first reported, for Permalloy, by Puchalska. ${ }^{28}$ The author showed that the SSD structure appears when the sample is deposited at an incidence angle above $45^{\circ}$, whereas the WSD structure can be observed for all angles. However, this is not the case for our material because (i) at normal incidence and if a perpendicular anisotropy is induced, we observe only the WSD structure, and (ii) at oblique incidence and below the critical angle, we observe neither the WSD nor the SSD structure, regardless of the thickness of the sample.

From the energetic point of view, the critical angle of deposition does not correspond directly to a critical tilt of the anisotropy, though we need a minimum of tilt to distinguish between the two structures. We shall rather consider the critical angle from the point of view of the anisotropies induced. In fact, when the angle of deposition is not zero, the oblique anisotropy $K_{o}$ appears and the existence of stripe domains is energetically favorable if the film is thick enough. But the oblique deposition also induces the
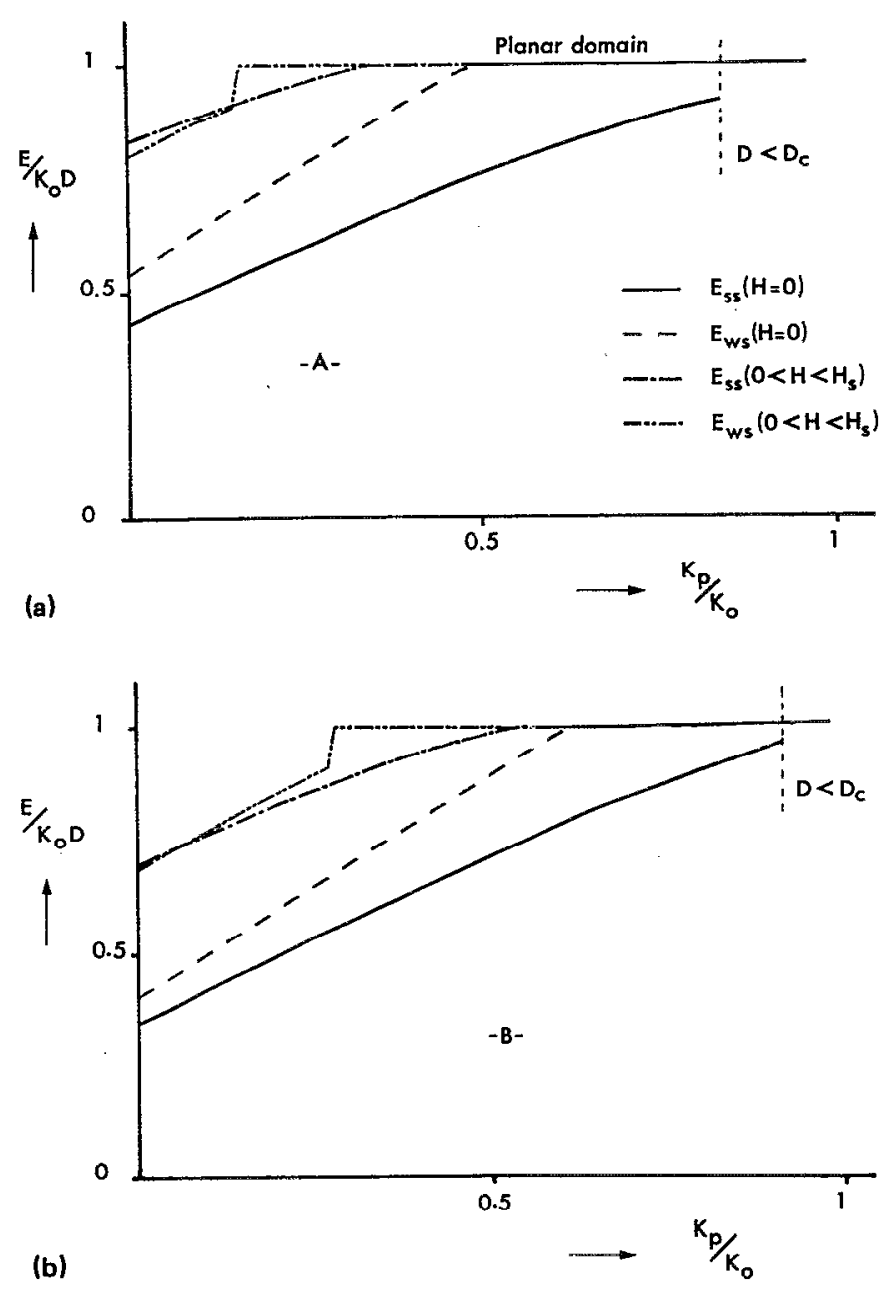

FIG. 10. (a) Numerical computation of the energy of stripe domain structures vs the ratio $K_{p} / K_{0}$ for $\mathrm{Ni}_{0.8} \mathrm{Co}_{0.2}$ films $\left(K_{o}=3 \times 10^{5} \mathrm{erg} / \mathrm{cc}\right.$ and $\left.\beta_{o}=20^{\circ}\right)$ and $\mathrm{Ni}_{0.5} \mathrm{Co}_{0.5}$ films $\left(K_{n}=5 \times 10^{5} \mathrm{erg} / \mathrm{cc}\right.$ and $\left.\beta_{o}=20^{\circ}\right)$. $E_{\mathrm{SS}}$ and $E_{\mathrm{WS}}$ represent the energy of SSD and WSD structures, respectively. The plot of these energies for an applied field $0<H<H_{s}$, where $H_{s}$ is the saturation field, indicates the range of $K_{p} / K_{o}$, where the transition from SSD to WSD structure is possible. (b) Calculated energy curves for $\mathrm{Ni}_{0.2} \mathrm{Co}_{0.8}$ films $\left(K_{o}=2 \times 10^{6} \mathrm{erg} / \mathrm{cc}\right.$ and $\left.\beta_{o}=20^{\circ}\right)$.

in-plane anisotropy $K_{p}$, which does not favor the stripes if the ratio $K_{p} / K_{v}$ is high. We shall return to formula (15), which gives the critical ratio $\left(K_{p} / K_{o}\right)_{c}$ and from which we can define the critical angle of deposition $\alpha_{c}$ as follows: (i)

TABLE II. Calculated values of $K_{p} / K_{o}$, obtained from Figs. 10(a) and $10(b)$, for which the transition from strong to weak stripe domains is possible. These values are compared with the experimental observations.

\begin{tabular}{lccc}
\hline \hline $\begin{array}{l}\text { Composition } \\
\text { Incidence angle }\end{array}$ & $\begin{array}{c}\mathrm{Ni}_{0.8} \mathrm{Co}_{0.2} \\
\alpha=40^{\circ}\end{array}$ & $\begin{array}{c}\mathrm{Ni}_{0.5} \mathrm{Co}_{0.5} \\
\alpha=40^{\circ}\end{array}$ & $\begin{array}{c}\mathrm{Ni}_{0.2} \mathrm{Co}_{0.8} \\
\alpha=60^{\circ}\end{array}$ \\
\hline $\begin{array}{l}\text { Transition possible } \\
\text { for } K_{p} / K_{\text {o }} \text { less than }\end{array}$ & 0.14 & 0.14 & 0.05 \\
$\begin{array}{l}\text { Measured values } \\
\text { of } K_{p} / K_{0}\end{array}$ & 0.01 to 0.2 & 0.18 to 0.3 & 0.24 to 0.4 \\
$\begin{array}{l}\text { Observed stripes } \\
\text { weak and } \\
\text { strong }\end{array}$ & $\begin{array}{c}\text { strong } \\
\text { only }\end{array}$ & $\begin{array}{c}\text { strong } \\
\text { only }\end{array}$ \\
\hline \hline
\end{tabular}


TABLE III. Experimental critical angle for existence of stripe domains in $\mathrm{Ni}_{x} \mathrm{Co}_{1-x}$ films.

\begin{tabular}{lccc}
\hline \hline Composition & $\mathrm{Ni}_{0.8} \mathrm{Co}_{0.2}$ & $\mathrm{Ni}_{0.5} \mathrm{Co}_{0.5}$ & $\mathrm{Ni}_{0.2} \mathrm{Co}_{0.8}$ \\
\hline Critical angle $\alpha_{c}$ & $<20^{\circ}$ & $40^{\circ} \pm 5^{\circ}$ & $60^{\circ} \pm 5^{\circ}$ \\
\hline \hline
\end{tabular}

For $0<\alpha<\alpha_{c}, K_{p} / K_{o}>\left(K_{p} / K_{o}\right)_{c}$, the stripe domains do not exist, and (ii) for $\alpha>\alpha_{c}, K_{p} / K_{o}<\left(K_{p} / K_{o}\right)_{c}$, the stripe domains exist.

It should be noted that the critical ratio $\left(K_{p} / K_{o}\right)_{c}$ approaches 1 for an infinite thickness. Let us see how this definition correlates with our experimental results (see Table III).

(1) For $\mathrm{Ni}_{0.8} \mathrm{Co}_{0.2}$ we cannot define a critical angle since the oblique anisotropy $K_{o}$, as we showed experimentally [Fig. 2(a)], is higher compared to the in-plane anisotropy $K_{p}$ (Fig. 4 ) because of the stress contribution. Thus the ratio $K_{p} / K_{o}$ is very small at any angle of deposition, and the stripe domains are expected to exist. We have confirmed this expectation down to an angle of incidence of $20^{\circ}$ (samples were not prepared at lower angles).

(2) The tensile stress found for low-incidence angles should not depend on the composition. Therefore, in the $\mathrm{Ni}_{0.5} \mathrm{Co}_{0.5}$ and $\mathrm{Ni}_{0.2} \mathrm{Co}_{0.8}$ films, the stress contributes to increase the in-plane anisotropy because of the positive magnetostriction of these alloys. Considering the value cxpected for $K_{o}$ at low-incidence angles, the ratio $K_{p} / K_{o}$ is then too high to allow the existence of the stripes. As the angle of deposition increases, the ratio decreases with decreasing stress and we expect that the critical angle of deposition will correspond to the angle for which the stress vanishes. This could give a qualitative explanation of the values found experimentally. For example, Fig. 11 shows a plot of the calculated values of the ratio $K_{p} / K_{o}$ versus the angle of deposition in case of the $\mathrm{Ni}_{0.5} \mathrm{Co}_{0.5}$ alloy. $\mathrm{Co}_{50} \mathrm{Ni}_{50}$ films were prepared only at $40^{\circ}$ and $60^{\circ}$. The curve of $\left(K_{p} / K_{o}\right)$ presented in Fig. 11 is a result of fitting a theoretical model with these measurements. The fit was performed assuming that (a) the crystal anisotropy constant

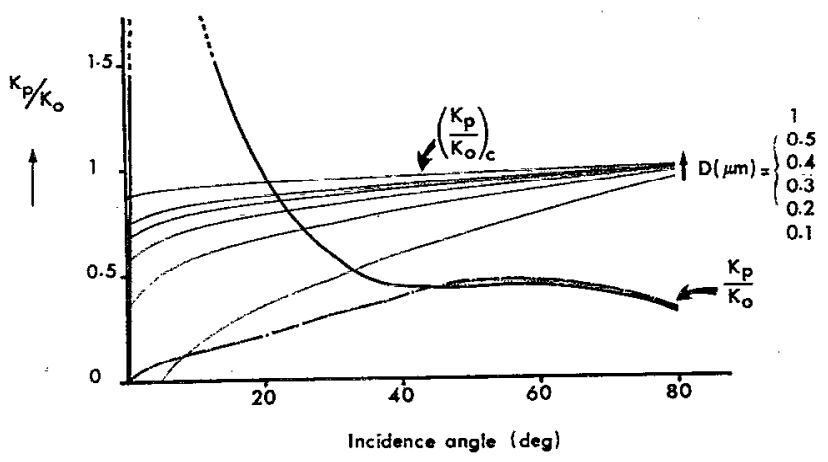

FIG. 11. Theoretical variation of the ratio $K_{p} / K_{v}$ with incidence angle for $\mathrm{Ni}_{0.5} \mathrm{Co}_{0.5}$ films. The ratio is compared to the critical ratio $\left(K_{p} / K_{o}\right)_{c}$, calculated for different thicknesses, in order to define the critical angle of incidence $\alpha_{c}$ The dashed line represents the variation of $K_{p} / K_{o}$ for shape anisotropies only. has the same value as for bulk material and (b) the relation between column dimensions and incidence angle is proportional to the values found for $\mathrm{Co}_{0.8} \mathrm{Ni}_{0.2}$. These calculated values of $K_{o}$ and $K_{p}$ are compared, in the same figure, to the critical ratio $\left(K_{p} / K_{o}\right)_{c}$ in order to define the critical angle of deposition is expected if we consider only shape anisotropies.

\section{v. CONCLUSIONS}

We have confirmed the effect of columnar grain morphology, related to the oblique deposition, on induced oblique and in-plane anisotropies in $\mathrm{Ni}_{x} \mathrm{Co}_{1-x}$ films. However, this effect is not only due to the shape of columns since the experimental results do not correlate with the values expected from shape anisotropy.

(1) In $\mathrm{Ni}_{0.8} \mathrm{Co}_{0.2}$ films, a tensile stress coupled with the negative magnetostriction increases the total anisotropy at low-incidence angles. For high angles of deposition, the stress vanishes and shape anisotropy predominates. The inclination of the oblique anisotropy axis is not affected by the stress and corresponds to the inclination of columns given by the tangent rule.

(2) In $\mathrm{Ni}_{0.2} \mathrm{Co}_{0.8}$ films the presence of a $c$-axis texture, due to the high precentage of cobalt, affects the direction and value of oblique anisotropy. At $60^{\circ}$ of incidence, the inclination of the oblique anisotropy axis corresponds to half the inclination expected from tangent rule for shape anisotropy alone.

Numerical calculations of strong and weak stripe-domain energies show the effect of the anisotropies on the existence and nature of these structures. The ratio $K_{p} / K_{o}$ seems to be the main factor to consider in this case, regardless of the thickness. The effect of the incidence angle and composition of the film are both related to this ratio.

\section{ACKNOWLEDGMENT}

This research was supported by the Community Action on Magnetic Storage Technology (CAMST) program.

${ }^{1}$ D. O. Smith, M. S. Cohen, and G. P. Weiss, J. Appl. Phys. 31, 1755 (1960).

${ }^{2}$ M. S. Cohen, J. Appl. Phys. 32, 87S (1961).

${ }^{3}$ K. Hara, J. Sci. Hiroschima Univ. Ser. II 34, 139 (1970).

${ }^{4}$ H. M. Ho, G. J. S. Gau, and G. Thomas, J. Appl. Phys. 65, 3161 (1989).

${ }^{5}$ E. I. Kondorsky and P. P. Denisov, IEEE Trans. Magn. MAG-6, 167 (1970).

${ }^{6}$ E. W. Pugh, J. Matisoo, D. E. Speliotis, and E. L. Boyd, J. Appl. Phys. 31, 239S (1960).

${ }^{7}$ I. B. Puchalska and R. P. Ferrier, Thin Solid Films 1, 437 (1968).

${ }^{8}$ E. Tatsumoto, K. Hara, and T. Hashimoto, Jpn. J. Appl. Phys. 7, 176 (1968).

${ }^{9}$ I. B. Puchalska, A. Hubert, S. Winkler, and B. Mirecki, IEEE Trans. Magn. MAG-24, 1787 (1988)

${ }^{10}$ H. Aitlamine, M. Labrune, and I. B. Puchalska, IEEE Trans. Magn. MAG-26, 48 (1990)

${ }^{11}$ K. Hara, M. Kamiya, K. Haschimoto, K. Okamoto, and H. Fujiwara, Thin Solid Films 158, 239 (1988).

${ }^{12}$ H. Fujiwara, Thin Solid Films 163, 379 (1988).

${ }^{13} \mathrm{H}$. Fujiwara, K. Hara, M. Kamiya, T. Hashimoto, and K. Okamoto, Thin Solid Films 163, 387 (1988).

${ }^{14}$ T. Daniel, thesis, University of Paris sud Orsay, 1985.

${ }^{15}$ E. Münster, J. Magn. Magn. Mater. 92, 279 (1990). 
${ }^{16} \mathrm{~J}$. M. Nieuwenhuizen and H. B. Haanstra, Philips Tech. Rev. 27, 87 (1966).

${ }^{17}$ J. W. Shih, Phys. Rev. 50, 376 (1936).

${ }^{18}$ L. W. McKeehan, Phys. Rev. 51, 136 (1937).

${ }^{19}$ M. Yamamoto and T. Nakamichi, J. Phys. Soc. Jpn. 2, 228 (1958).

${ }^{20}$ H. P. Murbach and H. Wilman, Proc. Phys. Soc. (London) B 66, 905 (1953).

${ }^{21}$ H. Wilman, Proc. Phys. Soc. (London) B 68, 474 (1955).
${ }^{22}$ I. W. Wolf, J. Appl. Phys. 33, 1152S (1962).

${ }^{23}$ G. P. Weiss and D. O. Smith, J. Appl. Phys. 3, 1166S (1962).

${ }^{24}$ J. D. Finegan and R. W. Hoffman, J. A.ppl. Phys. 30, 597 (1959)

${ }^{25}$ M. Labrune and J. Miltat, IEEE Trans. Magn. MAG-26, 1521 (1990).

${ }^{26}$ A. Hubert, IEEE Trans. Magn. MAG-21, 1604 (1985).

${ }^{27}$ A. Hubert (private communication).

${ }^{28}$ I. B. Puchalska, Acta Phys. Pol. 36, 589 (1969). 